\title{
Adhesion and friction in hard and soft contacts: theory and experiment
}

\author{
Valentin L. POPOV ${ }^{1,2, *}$, Qiang LI ${ }^{1, *}$, Iakov A. LYASHENKO ${ }^{1,3}$, Roman POHRT ${ }^{1}$ \\ ${ }^{1}$ Technische Universität Berlin, Berlin 10623, Germany \\ ${ }^{2}$ National Research Tomsk State University, Tomsk 634050, Russia \\ ${ }^{3}$ Sumy State University, Sumy 40007, Ukraine \\ Received: 02 June 2020 / Revised: 14 October2020 / Accepted: 10 December 2020 \\ (C) The author(s) 2020 .
}

\begin{abstract}
This paper is devoted to an analytical, numerical, and experimental analysis of adhesive contacts subjected to tangential motion. In particular, it addresses the phenomenon of instable, jerky movement of the boundary of the adhesive contact zone and its dependence on the surface roughness. We argue that the "adhesion instabilities" with instable movements of the contact boundary cause energy dissipation similarly to the elastic instabilities mechanism. This leads to different effective works of adhesion when the contact area expands and contracts. This effect is interpreted in terms of "friction" to the movement of the contact boundary. We consider two main contributions to friction: (a) boundary line contribution and (b) area contribution. In normal and rolling contacts, the only contribution is due to the boundary friction, while in sliding both contributions may be present. The boundary contribution prevails in very small, smooth, and hard contacts (as e.g., diamond-like-carbon (DLC) coatings), while the area contribution is prevailing in large soft contacts. Simulations suggest that the friction due to adhesion instabilities is governed by "Johnson parameter". Experiments suggest that for soft bodies like rubber, the stresses in the contact area can be characterized by a constant critical value. Experiments were carried out using a setup allowing for observing the contact area with a camera placed under a soft transparent rubber layer. Soft contacts show a great variety of instabilities when sliding with low velocity - depending on the indentation depth and the shape of the contacting bodies. These instabilities can be classified as "microscopic" caused by the roughness or chemical inhomogeneity of the surfaces and "macroscopic" which appear also in smooth contacts. The latter may be related to interface waves which are observed in large contacts or at small indentation depths. Numerical simulations were performed using the Boundary Element Method (BEM).
\end{abstract}

Keywords: adhesion; friction; adhesion hysteresis; Boundary Element Method (BEM); hard solids; soft matter

\section{Introduction}

Since the famous work by Johnson, Kendall, and Roberts (JKR) from 1971 [1], adhesive contacts have remained in focus of research in contact mechanics and tribology. In the JKR theory, the action range of adhesive forces is assumed to be zero (or much smaller than any characteristic length of contact). In 1975, Derjaguin, Muller, and Toporov (DMT) suggested a model in which the final interaction range was considered explicitly (in particular, the interaction outside the contact

* Corresponding authors: Valentin L.POPOV, E-mail: v.popov@tu-berlin.de; Qiang LI, E-mail: qiang.li@tu-berlin.de 
area) [2]. Tabor solved in 1977 the controversy between both theories stating clearly that the JKR and DMT theories are limiting cases for very small and very large range of action of adhesive forces and introduced a parameter (now known as Tabor parameter) which describes transition between these two limiting cases [3]. In the early 2000s, the interest in adhesive contacts was driven by studying adhesion in biological adhesion "devices" like in gecko feet [4] or other biological structures [5]. In the last years, adhesive contacts have become again a hot topic, in particular in the context of adhesion of functionally graded materials [6], the loading-unloading hysteresis in rough contacts [7] as well as adhesive contacts under tangential loading [8]. In particular, rough contacts have been very intensively studied, for example, evaluation of effective adhesion work based on the Maugis-Dugdale model [9, 10], description of rough surfaces and development of experimental methods [11].

Two developments of the last years greatly facilitated the advancement in adhesive contact's research:

1) The development of the Fast-Fourier-Transformation-assisted Boundary Element Method (FFTassisted BEM) [12-14], in particular, its adaptation for simulation of adhesive contacts [15].

2) The development of experimental methods for direct observation of the processes of attachment and detachment $[16,17]$.

One of the striking experimental findings are complicated stick-slip dynamics of tangential adhesive contacts [17]. These are far from being understood well and are subject of intense debates [18-20].

The present paper is devoted to an analytical, numerical, and experimental investigation of adhesive contacts under tangential loading and rolling. However, the dissipative properties of normal adhesive contacts are also considered as far as this helps understanding friction.

For slow sliding of adhesive contacts, the application of some force is needed, which can be interpreted as force of friction. There are two main generic mechanisms of friction in adhesive contacts: (a) Either the friction in the boundary line - due to its unstable sliding as described in Ref. [21], or (b) the friction directly in the inner part of the contact area $[8,22]$. In the present paper, both mechanisms are investigated analytically and numerically. For the former one, the numerical study is conducted by use of the BEM for the JKR-type adhesive contact. The analysis is restricted to the elastic contact under very slow normal and tangential movement (quasi-static contact), so that viscoelastic and inertia properties can be neglected.

We will consider both basic types of friction due to relative movement of bodies: rolling and sliding friction. These types have important differences stemming from the different local direction of movement of surfaces. Pure rolling is essentially a normal contact problem, because the surfaces at the leading edge are approaching each other in the normal direction and on the rear edge they separate in normal direction, both without any tangential movement. It is important to note that the absence of relative tangential movement of surfaces in the case of a rolling contact suppresses the contribution from shearing of the contact area, while in a sliding contact this contribution not only exists but presumably represents the main contribution to friction in most cases.

The structure of the paper reflects this understanding.

The first part of the paper is devoted to numerical simulation of adhesive contacts. Section 2 reports results on adhesive hysteresis in normal and rolling contacts. Section 3 considers sliding adhesive contacts with both boundary and area contributions.

The second part of the paper is devoted to experimental investigation on the contacts of rigid indenters and soft rubber. Reported are results for normal contacts and sliding.

\section{Normal and rolling contacts: numerical simulation}

The solution of Johnson, Kendall, and Roberts (JKR) [1] assumes that the tangential stresses in the contact area vanish. For ideally smooth surfaces, this assumption is a logical consequence of the independence of the potential energy of an adhesive contact on its lateral position. Real adhesive contacts, on the contrary, typically show very high 
friction, which physically is caused by microscopic heterogeneities. However, there exists a class of adhesive contacts in which the friction in the contact area could be small. To have this property, the contacting bodies have to be free of viscosity, plasticity and should not show elastic instabilities (which, according to Prandtl, are the main mechanism of energy dissipation in purely elastic systems [23-25].) Under these conditions, the surfaces would be in a state of "structural superlubricity" as described in [26-28]. The tangential friction in the contact area does not appear also in the pure normal and rolling contacts. We therefore start with this class of dynamic contacts, focusing our attention first on the boundary contribution to energy dissipation.

\subsection{Methodology}

Numerical simulations presented in Section 2 were carried out for adhesive contact of a parabolic indenter with the radius of curvature $R$ superimposed with a two-dimensional waviness with amplitude $h$ and wave length $\lambda$ :

$$
z(x, y)=\frac{x^{2}+y^{2}}{2 R}+h \cdot \sin \left(\frac{2 \pi}{\lambda} x\right) \cdot \sin \left(\frac{2 \pi}{\lambda} y\right)
$$

see subplot of Fig. 1(b) for an example. In the following subsections, simulations were performed using the FFT-assisted BEM [29] under displacementcontrolled conditions and with the same assumptions as in JKR theory: The materials behave as linear elastic half-spaces with surface slopes being low and adhesion only acts in the regions of intimate contact. Validation of this method was provided in [15, 29-31]. Typically, a grid with $512 \times 512$ points was used.

\subsection{Normal adhesive contact of rough surfaces}

Contrary to a non-adhesive contact of elastic bodies, an adhesive contact has intrinsic dissipative properties, which can best be seen in a complete cycle of formation and destruction of contact. The area between the indentation and detachment branches of force-displacement relation (e.g., in the JKR theory) is the dissipated energy per cycle. The JKR theory is based on the principle of virtual work, meaning
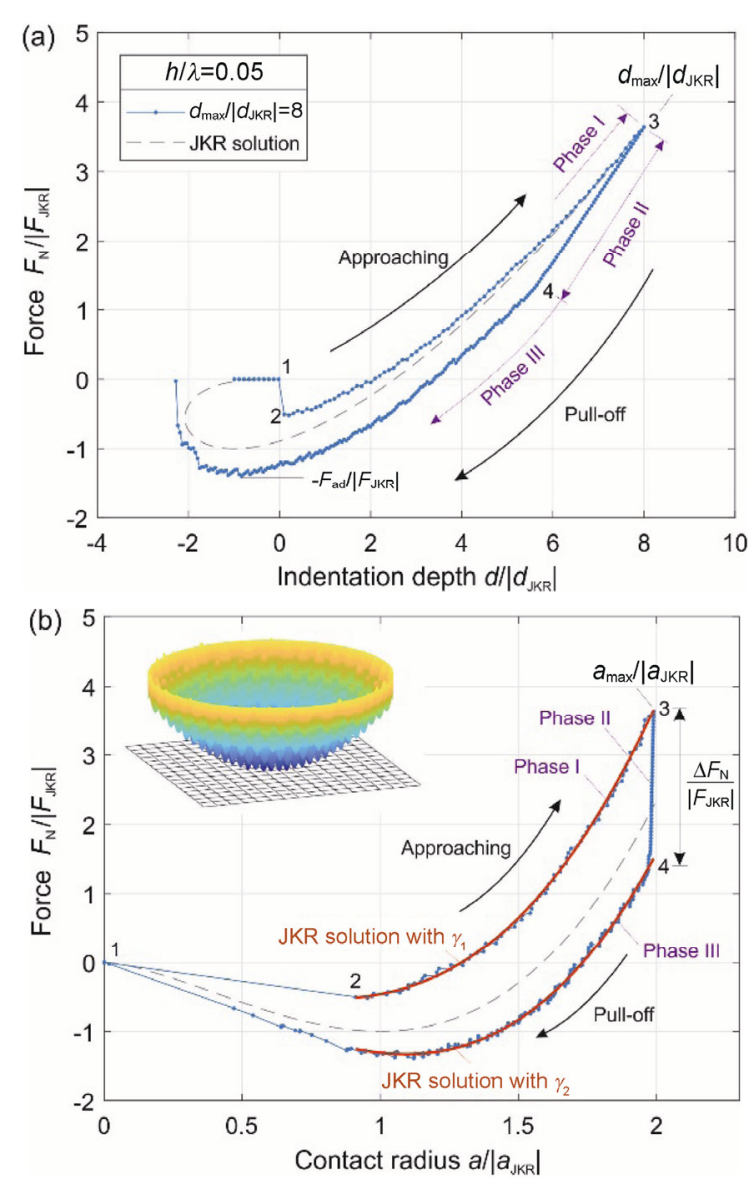

Fig. 1 Simulation of an adhesive indentation test using a parbolic indenter with waviness according to (1) and $h / \lambda=0.05$. (a) Force-distance and (b) force-contact radius dependencies during indenting and pull-off stages. The contact area remains constant immediately after turning from the indentation to the pull-off (phase II). The gray dashed curve is the JKR solution without waviness.

that adhesive contact is reversible in all phases when static equilibrium is possible [32]. Irreversible energy dissipation occurs only during the instable jumps from one equilibrium state to the other. This occurs in the moments of coming into contact and in the sudden destruction of contact. Independently from whether such jumps occur during a normal or a tangential movement, energy is dissipated. We therefore start in this Section with discussion of dissipation in the normal adhesive contacts.

According to the JKR theory, the system jumps into contact and then moves forth and back on the same curve. The situation changes for more complicated shapes. Even for flat-ended stamps with compact face shape, the detachment may occur in a series of 
consecutive instabilities [15]. The same is valid when surfaces are rough. Our numerical simulations show that during approach and detachment, the movement of the contact boundary proceeds in both continuous changes and instable jumps. Each jump is irreversible and, in its course, energy is lost. Due to the multiple microscopic instabilities, the indentation differs from the detachment curve at all values of the indentation depth: the dissipation leads to an apparent "friction" counteracting the movement of the contact boundary.

Figure 1 shows the results of a simulation of the indentation and detachment of a parabolic indenter with superposed waviness into an elastic halfspace. The above mentioned instabilities are clearly visible in the curves in Fig. 1 as microstructure of the lines. When observing the evolution of the contact zone, the corresponding local instabilities can be clearly identified.

In the following analysis, the normal force $F_{\mathrm{N}}$, the indentation depth $d$, and the contact radius $a$ will be normalized by the critical values of JKR solution for a smooth sphere $[1,33]$ :

$$
\begin{gathered}
F_{\mathrm{JKR}}=-\frac{3}{2} \pi \gamma_{0} R \\
d_{\mathrm{JKR}}=-\left(\frac{3 \pi^{2} \gamma_{0}^{2} R}{64 E^{* 2}}\right)^{1 / 3} \\
a_{\mathrm{JKR}}=\left(\frac{9 \pi \gamma_{0} R^{2}}{8 E^{*}}\right)^{1 / 3}
\end{gathered}
$$

where $\gamma_{0}$ is the work of adhesion per unit area and $E^{*}=E /\left(1-v^{2}\right)$ is the effective elastic modulus of a half-space with Young's modulus $E$ and Poisson's ratio $v$.

In 1995, Johnson studied the adhesive contact between a wavy surface and a half-space [34], and showed that the distinction between rough and smooth surfaces is governed by the parameter, which we now call the "Johnson parameter":

$$
\alpha=\sqrt{\frac{2 \lambda \gamma_{0}}{\pi^{2} h^{2} E^{*}}}
$$

In the following sections, we show that the Johnson parameter is an essential governing parameter for both adhesion and friction.

\subsection{Effective surface energies for shrinking and expanding of adhesive contacts}

Figure 1 represents typical simulation results for the force-indentation and force-contact area relations. Since the contact boundary is generally noncircular, we define the contact radius as the distance from the center of contact to the furthest remote contact point, independently of whether the contact area is compact or consists of a "cloud of contact spots" as shown in the right hand side of Fig. 2(b).

Figure 1 allows to explain general features which are important for the following analysis:

1) Both indentation and pull-off curves follow very closely the JKR solutions, shown in Fig. 1(b) with red lines, but with different values for the specific work of adhesion. The value $\gamma_{1}$ for the approach is smaller than $\gamma_{2}$ for pull-off. See Section 2.5 for limitations of this simple picture with respect to very large roughness values.

2) When the direction of loading changes from

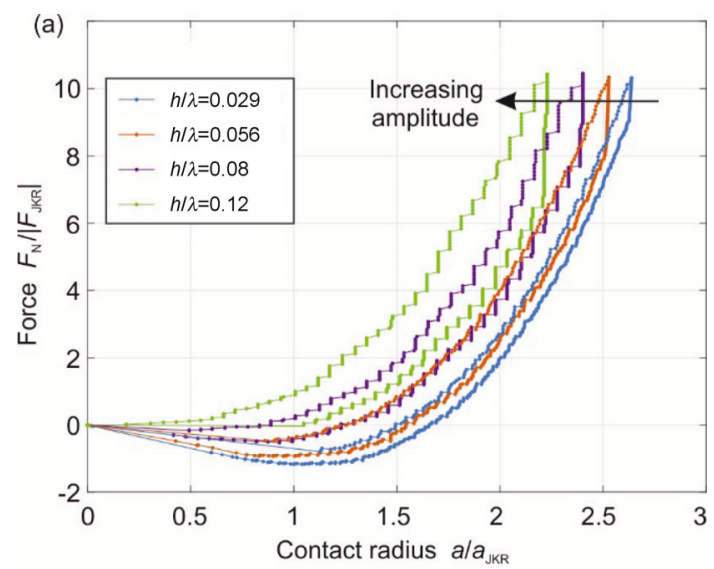

(b)

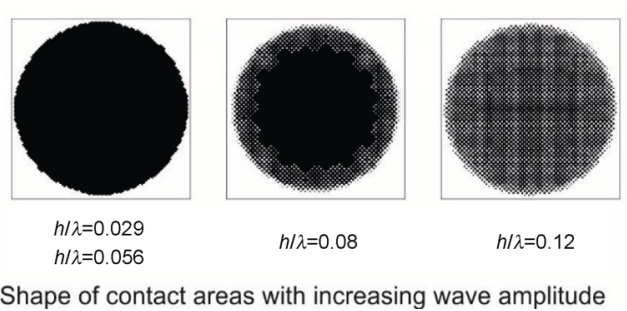

Fig. 2 (a) Dependence of the force on the contact radius, for four different roughness amplitudes $h / \lambda$, varying from 0.029 to 0.12 , and for the same maximal indentation depth $d_{\max } /$ $\left|d_{\mathrm{JKR}}\right|=15$. (b) Shapes of contact areas for different roughness amplitudes. 
approach to pull-off, the contact area remains constant for a while. This feature is seen very clearly in the dependence of force on contact radius in Fig. 1(b) or Fig. 2(a). The dependence of force on the indentation depth is linear in this phase. This behavior is as if a force of static friction acted on the boundary line, keeping it in place when the motion of the indentation depth is reversed. A similar phenomenon is theoretically described in Ref. [35] where the effect is caused by microscopic chemical heterogeneities.

The above findings mean that the normal adhesive contact of rough surfaces can be characterized by effective energies for approach and pull-off, in other words for the expansion and retraction of the contact zone. The values of the effective energies are expected to approach zero for very rough surfaces and roughly the microscopic value of the specific work of adhesion $\gamma_{0}$ for smooth surfaces.

Figure 3 shows the results of systematic parametric studies of effective specific surface energies $\gamma_{1}$ and $\gamma_{2}$. All data points collapse to well-defined master curves if the effective energies are plotted as function of the Johnson parameter [36]. In most simulations, all parameters have been fixed and only the roughness amplitude was varied. These results are plotted in Fig. 3 with gray squares. However, we also performed a large number of simulations in which the elastic modulus, the roughness amplitude and wave-length, and the microscopic specific work of adhesion $\gamma_{0}$ were varied in a random fashion. These additional data points provide a proof of the hypothesis, that the true determining parameter is the Johnson parameter. They are shown in Fig. 3 with open triangles having different colors for different sets of parameters.

For the normalized effective surface energies $\gamma_{1} / \gamma_{0}$ and $\gamma_{2} / \gamma_{0}$, as well as the maximum force of adhesion $\left|F_{\text {ad }}\right| /\left|F_{\text {JKR }}\right|$, all data points collapse on a well identified dependencies, confirming that they depend indeed only on the Johnson parameter:

$$
\begin{gathered}
\gamma_{1} / \gamma_{0}=f_{1}(\alpha) \\
\gamma_{2} / \gamma_{0}=f_{2}(\alpha) \\
\left|F_{\text {ad }}\right| /\left|F_{\text {JKR }}\right|=\Phi(\alpha)
\end{gathered}
$$

Let us discuss the above dependencies in more detail. For large values of the Johnson parameter, all three dependencies tend towards 1 . This is expected, since higher values of $\alpha$ correspond to small roughness amplitude, thus approaching an ideally smooth surface.

An increase of the roughness amplitude corresponds to a decrease of the Johnson parameter. The effective specific work of adhesion describing the indentation phase $\gamma_{1}$, is continuously decreasing together with the Johnson parameter. A particularly sharp drop to almost zero occurs in the vicinity of $\alpha=0.5$. A closer analysis of the contact configuration shows that this sharp drop is associated with the change from the compact contact area to a cloud of separated contact spots. This reaffirms the conclusions from the original paper by Johnson [34]. For $\alpha>0.5$, the contact area has a relatively well-defined outer border, and the force-distance dependence can be accurately described by the regular JKR theory
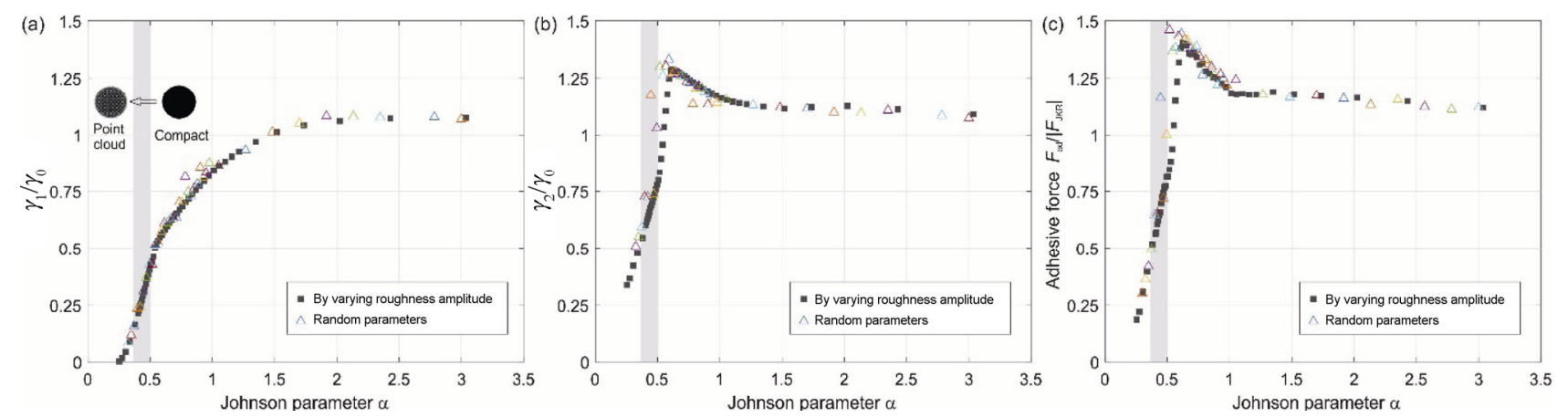

Fig. 3 Dependence on Johnson parameter of the effective specific work of (a) adhesion $\gamma_{1}$ for approach, (b) $\gamma_{2}$ for detachment, and (c) the maximum adhesive force $F_{\text {ad }}$. The gray squares correspond to numerical results by varying only the roughness amplitude, and the triangles by randomly varied parameters (including roughness amplitude, elastic modulus and specific work of adhesion $\gamma_{0}$ ). In all cases, the wave length was kept small in comparison to the sphere radius $\lambda / R$ ranged from 0.005 to 0.0075 . 
with a modified specific work of adhesion. Surfaces with $\alpha>0.5$ are practically non-adhesive.

A slightly different behavior is found in the effective specific energy $\gamma_{2}$ determining the detachment process. A decrease of the Johnson parameter, or increase of roughness, first leads to an increase of the effective surface energy followed by an abrupt decrease, see also Ref. [36]. The maximum value is found at $\alpha=0.6073$. The force of adhesion is observed of course during the detachment process, so it is strongly correlated with $\gamma_{2}$, as for parabolic bodies, the specific work of adhesion directly determines the force of adhesion $F_{\mathrm{ad}}=\frac{3}{2} \pi R \gamma_{2}$.

\subsection{Influence of size effects}

In Fig. 1, the instabilities due to roughness are seen as small fluctuations of the indentation and pull-off curves. In some applications like the contact between a cell and the tip of an atomic force microscope, the contact radius can be very small. When it is comparable with the scale of the roughness, the microstructure becomes pronounced, as exemplified in Fig. 4. The observable jumps are sometimes interpreted as results of detachment of discrete adhesive bonds [37], but our simulations suggest, that this kind of discontinuity can also be due to the roughness of the indenter or the soft body studied.

\subsection{Friction of the contact boundary}

Note that the above simple picture of two effective surface energies works well only when amplitude of roughness is not too large. With reference to the

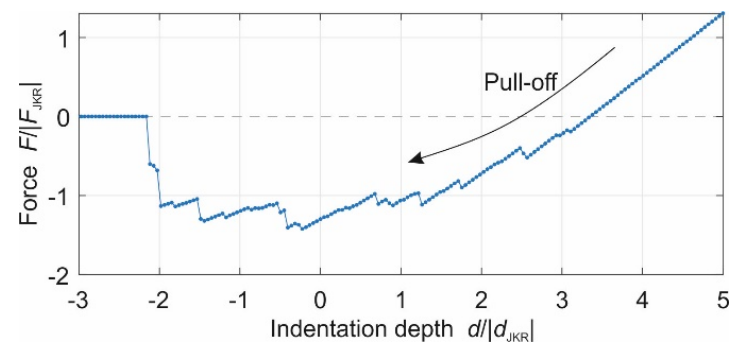

Fig. 4 An exemplary dependence of the normal force on the indentation depth for a spherical indenter with a superimposed roughness of the form (1) in the case when the scale of roughness approaches the characteristic size of the contact. Note that the instable detachments are distributed irregularly despite of the regular waviness of the indenter. first of pictures in Fig. 2(b), one can state that this concept is applicable with reasonable accuracy to contacts which are compact or at least mainly compact, which corresponds to $\alpha>0.5$. It is not applicable to the regimes when the contact consists of a cloud of separate points, which happens for $\alpha<0.5$. This can be seen from the leftmost curve of Fig. 2(a): in this case, the force of adhesion practically vanishes. However, the indentation and detachment curves do not coincide. On the contrary, they show an even bigger hysteresis compared with smaller amplitudes of roughness. For $\alpha<0.5$ neither the indentation nor the detachment is well-described by JKR theory.

For now, let us concentrate our attention on the range $\alpha>0.6$. This is the interval in which the contact area possesses a well-defined boundary, even when it is not exactly circular, but represents a "rough line" or even consists of a narrow band of separated contact spots. On the other hand, we see from Fig. 5 that in this interval, the mean value of the effective surface energies, $\left(\gamma_{1}+\gamma_{2}\right) / 2$, is roughly constant and equal to its microscopic value $\gamma_{0}$. The effective surface energies for indentation and detachment can thus be approximately written as

$$
\begin{aligned}
& \gamma_{1}=\frac{1}{2}\left(\gamma_{1}+\gamma_{2}\right)-\frac{1}{2}\left(\gamma_{2}-\gamma_{1}\right) \approx \gamma_{0}-\Delta \gamma / 2 \\
& \gamma_{2}=\frac{1}{2}\left(\gamma_{1}+\gamma_{2}\right)+\frac{1}{2}\left(\gamma_{2}-\gamma_{1}\right) \approx \gamma_{0}+\Delta \gamma / 2
\end{aligned}
$$

with

$$
\Delta \gamma=\gamma_{2}-\gamma_{1}
$$

It is well-known that the specific surface energy can be interpreted as the linear force density acting

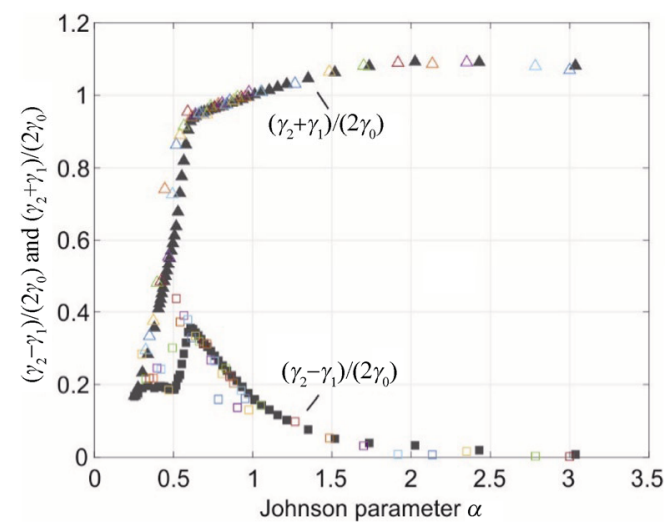

Fig. 5 Dependence of $\left(\gamma_{1}-\gamma_{2}\right) /\left(2 \gamma_{0}\right)$ and $\left(\gamma_{1} \gamma_{2}\right) /\left(2 \gamma_{0}\right)$ on the Johnson parameter. 
on the boundary line. We explain it [38] with an example of a soap film stretched within a squareshaped wire frame with length $l$. The work done by the external force $F$ for a displacement $\Delta x$ is $W_{\mathrm{e}}=$ $F \Delta x$, the surface area is increased by $l \Delta x$, and the surface energy by $W_{\mathrm{s}}=2 \gamma_{0} l \Delta x$. Equilibrium of both gives that linear force density is equal to $F / l=$ $2 \gamma_{0}$. The force pro length for pulling on a movable side of the frame is twice of the surface energy of liquid [38]. For smooth surfaces in the studied case, this linear force density is equal to the specific surface energy $\gamma_{0}$. For rough surfaces, it depends on the direction of the movement of the boundary, increasing by $\Delta \gamma / 2$ for moving in one direction and decreasing by the same value for moving in the opposite direction. Thus, the quantity

$$
q=\frac{1}{2} \Delta \gamma
$$

can be interpreted as a linear density of the force of friction, acting always opposite to the motion direction of the contact boundary.

The dependence of the boundary friction on the Johnson parameter is also shown in Fig. 5. In contrast to the absolute values of the surface energies, their difference, and thus the boundary friction vanishes for both small and large roughness values and exhibits an extremum at the boundary of the considered interval, i.e., at $\alpha \approx 0.6$.

\subsection{Rolling adhesive contact}

During rolling, tangential movement of the contact area occurs due to superposition of approach at the front edge and detachment at the rear edge. Due to the adhesive hysteresis, the whole system dissipates energy and rolling friction emerges. The dissipated energy can directly be estimated analytically. As shown in Section 2.2, the closing of the contact and its opening can be characterized by two different specific surface energies $\gamma_{1}$ and $\gamma_{2}$. Consider an adhesive contact with contact radius $a$, which is moved tangentially by the distance $\Delta x$. During this movement, surface area $\Delta A=2 a \Delta \mathrm{x}$ will come into contact at the front side and the same area will detach at the back side. The net dissipated energy thus will be $\Delta W=\Delta A\left(\gamma_{2}-\gamma_{1}\right)=2 a\left(\gamma_{2}-\gamma_{1}\right) \Delta x$. The friction force can be defined as the ratio of the dissipated energy to the sliding distance:

$$
F_{\mathrm{T}}=2 a\left(\gamma_{2}-\gamma_{1}\right)
$$

If the rolling contact does not have an exact circular form, then the half-width of the contact in the direction perpendicular to the rolling direction should be used in Eq. (12) as the contact radius. We see that the adhesion contribution to rolling friction is indeed the friction of the contact boundary from Eq. (11) times the boundary width, twice (since both the leading and the trailing edge advance). A similar approach was used and experimentally confirmed by Kendall [39] for the rolling friction of cylindrical rollers.

Now let us consider results of numerical simulation of rolling spheres with the same type of waviness as defined by Eq. (1). Simulations have been carried out under condition of fixed indentation. As can be seen in Fig. 6(a), the normal force shows only very small fluctuations, so that there is no substantial

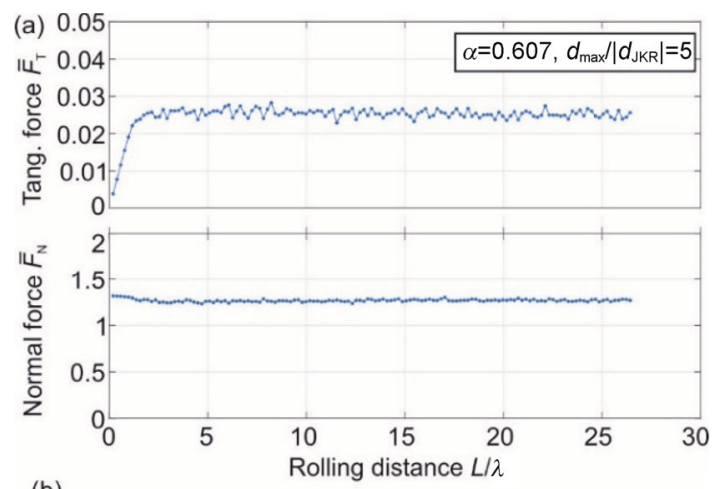

(b)

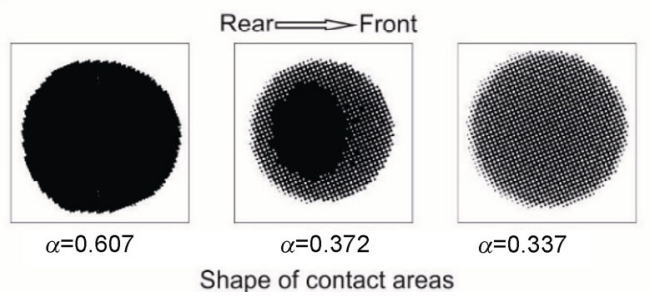

Fig. 6 An example of rolling of a rough rigid sphere on a smooth elastic half-space: (a) dependencies of the normal and tangential forces (normalized by the absolute value of Eq. (2)) on the rolling distance. (b) Typical contact shapes for different roughness amplitudes (characterized by the Johnson parameter $\alpha$ ). The first snapshot corresponds to the process shown in (a). It is seen that the contact shape is not circular, showing smaller radius (corresponding to smaller $\gamma$ ) on the leading edge, and a larger radius (corresponding to larger $\gamma$ ) on the trailing edge. 
difference between indentation control and force control. In the simulation for tangential contact, the indenter slides or rolls very slowly (statically), so at each moment, the adhesive normal contact solution obtained through BEM, then the tangential force was calculated as the integral over the contact area of the local pressure multiplied with the $x$-component of the gradient of the rigid surface. The tangential force shows considerable fluctuations caused by instabilities of the boundary line but these can still be characterized as "microscopic" so that the macroscopic force of friction can be identified easily. A snapshot of the contact configuration is shown in the first picture of Fig. 6(b). One can see that the boundary of the contact area is irregular and the macroscopic shape of the contact is not ideally circular.

To verify hypothesis Eq. (12), a large number simulations with varying parameters were carried out. Figure 7 shows values of the tangential force plotted against $2 a\left(\gamma_{2}-\gamma_{1}\right)$. Both $F_{\mathrm{T}}$ and $a$ were found numerically. For the values of Johnson parameter larger $\alpha=0.506$, all results collapse to a linear dependency with slope 1, thus indeed confirming Eq. (12). Note that this contribution to the force of rolling friction is proportional to the contact radius rather than to the contact area.

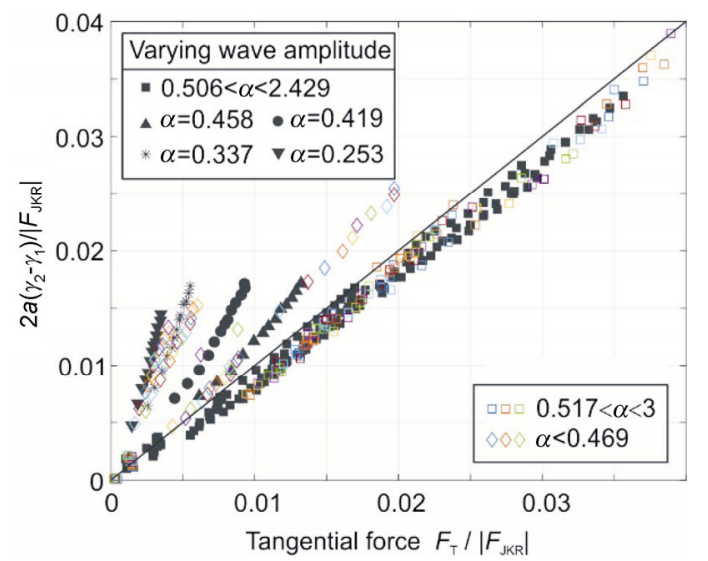

Fig. 7 Comparision of tangential force from numerical simulation with prediction of $2 a\left(\gamma_{2}-\gamma_{1}\right)$. For large enough Johnson parameters, for which the contact area is compact, the tangential force is equal to this value. Otherwise, the contact is non-compact in form of a cloud of contact spots and the tangential force is overestimated by Eq. (12). Numerical results have been obtained for 13 different indentation depths from $d_{\mathrm{max}} /\left|d_{\mathrm{JKR}}\right|=1-13$. Both the tangential force and $2 a$ $\left(\gamma_{2}-\gamma_{1}\right)$ are normalized by $F_{\mathrm{JKR}}$.
For smaller values of the Johnson parameter, as explained above, the concept of effective surface energies for opening and closing the adhesive crack cannot be applied. Thus, as expected, the data do not fit the linear dependence Eq. (12). Rolling resistance for this range of Johnson parameter was considered in Refs. [40-43].

Analytical estimation of the force of rolling friction according to Eq. (12) requires two specific surface energies and the contact radius. For determining the contact radius, one could argue that a rolling contact is a combination of indentation at the leading edge and detachment at the rear edge. Thus, in average, it should approximately correspond to a smooth contact with the true surface energy $\gamma_{0}$. Numerical simulations show that the contact radius of a rolling contact corresponds to an effective energy of about $\gamma_{3} \approx 1.15 \gamma_{0}$ (Fig. 8). Similar to normal contact, we use the JKR solution with another adhesion work $\gamma_{3}$ to approximate the tangential force-contact radius curve. The dependence of $\gamma_{3}$ on Johnson parameter is seen in Fig. 8. The contact radius thus can be obtained from the usual JKR relation:

$$
d=\frac{a^{2}}{R}-\sqrt{\frac{2 \pi \gamma_{3} a}{E^{*}}} \approx \frac{a^{2}}{R}-\sqrt{\frac{2 \pi a\left(1.15 \gamma_{0}\right)}{E^{*}}}
$$

\section{Sliding adhesive contact: numerical simulations}

For rolling, as for any other normal contact, it is of

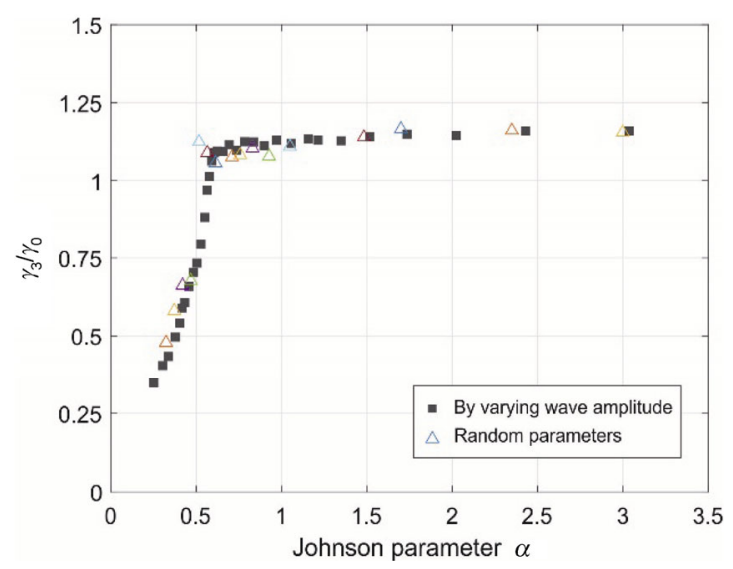

Fig. 8 Dependence of the effective specific work of adhesion, $\gamma_{3}$, determining the contact radius of a rolling contact, on the Johnson parameter. $\gamma_{3}$ was obtained using the JKR solution to approximate the dependence of the normal force on the contact radius. 
no importance whether the stiff or the elastic body is rough since only the relative (composed) roughness of both bodies plays a role. In contrast, the sliding contact is a purely tangential contact and it is important which of the bodies is rough. For example, in a contact of a rigid rough indenter and a smooth elastic half-space, the sliding force is zero (assuming of course the validity of presuppositions of the JKR theory). When a smooth indenter slides on a rough elastic surface, it is not zero. In the first case, the contact configuration remains unchanged all the time, in the second it generally changes and may also include instable configurations leading to dissipation and friction.

For numerical simulation of sliding friction, we considered a contact of a rough rigid sphere with a rough elastic half-space. The "roughness" of both bodies was modeled by the two-dimensional waviness Eq. (1) with identical $h$ and $\lambda$. The direction of the axes of waviness of two bodies were rotated relative to each to make the surfaces incommensurable. The simulation procedure was the same as in the case of rolling contact, only the changes of the interfacial gap were caused by relative sliding instead of rolling. As discussed in the introduction, the frictional force in adhesive sliding contacts may contain two parts: friction at the contact boundary $F_{\mathrm{B}}$, and a contribution of the inner contact area, $F_{\mathrm{C}}$ :

$$
F_{\text {Friction }}=F_{\mathrm{B}}+F_{\mathrm{C}}
$$

\subsection{Friction of the contact boundary}

As in the case of rolling, the dynamics of the contact configuration consists of both continuous movements of the boundary and instable jumps. The continuous movement is based on energy balance and is completely reversible. The jumps lead to energy dissipation and irreversibility of the tangential sliding, which is perceived macroscopically as the force of friction. This process is illustrated in Fig. 9 showing four snapshots of the contact configuration during sliding process. For this simulation, the wavelength of the roughness was chosen relatively large to visualize the irregularity of the boundary and its jerky behavior.

Figure 10 shows the dependence of the contribution to the friction force due to boundary jumps on
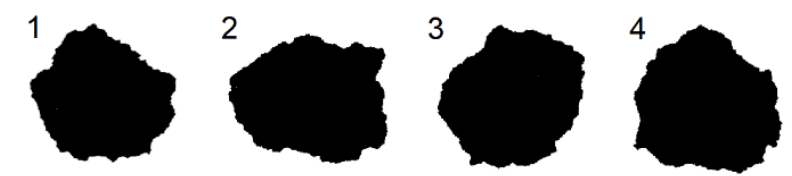

Fig. 9 Consecutive snapshots of the contact area during sliding. The dynamics of the contact configuration consists of the phases of continuous evolution and instable jumps.
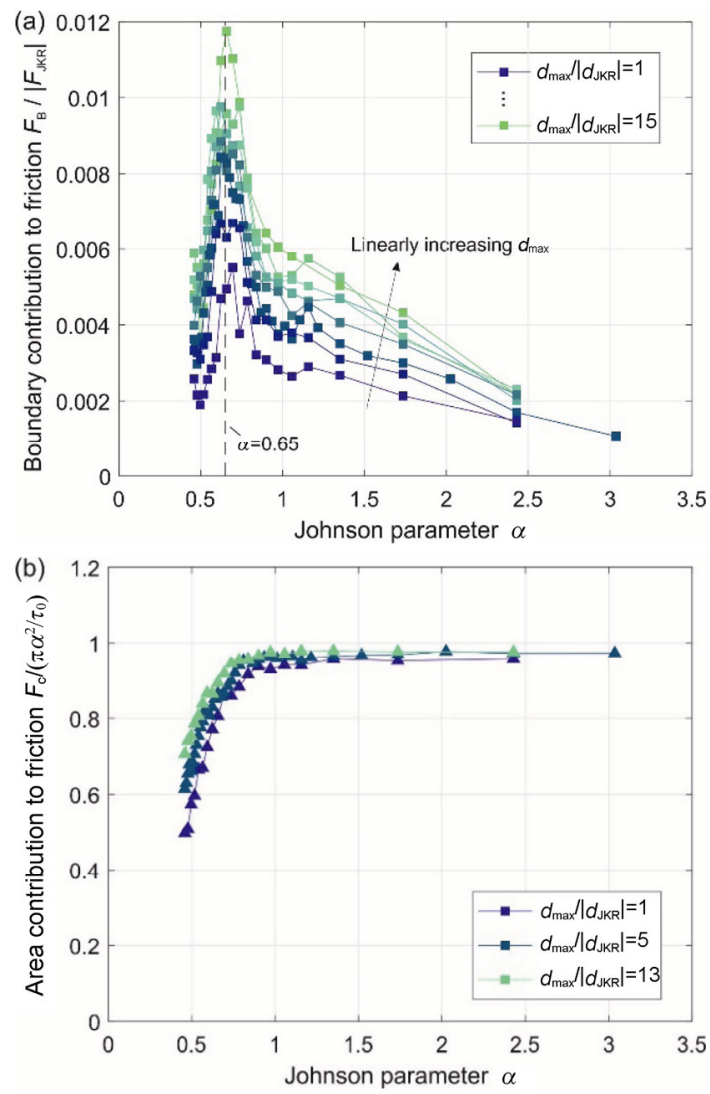

Fig. 10 (a) Dependence of the boundary contribution to the force of friction and (b) contact area contribution to the force of friction on Johnson parameter.

the Johnson parameter $\alpha$. The tangential force is small both in the limit of very smooth and very rough surfaces, and achieves a maximum exactly at the transition from smooth (adhesive) to rough (nonadhesive) surfaces, corresponding to $\alpha \approx 0.65$.

\subsection{Area contribution to friction}

The contribution from the area can be interpreted in different ways: either as a microscopic coefficient of friction to be multiplied with the adhesive pressure or as shearing of some physically existing boundary layer $[8,22]$. In the latter case, there exists some characteristic tangential stress $\tau_{0}$ to be 
overcome. The corresponding contribution to the tangential force during sliding is then proportional to the real contact area, $A$. For large Johnson parameters (small roughness), the contact area is compact and practically coincides with $\pi a^{2}$. The compactness of the contact, expressed as the numerically calculated value for the real contact area normalized by $\tau_{0} \pi a^{2}$ is shown in Fig. 10(b). For values of $\alpha<0.65$ approximately, it decays quickly. It is seen that at the large value of Johnson Parameter, the friction is roughly equal to the production of contact area and this characteristic tangential stress.

\section{Experimental setup}

The second part of the paper is devoted to an experimental study of adhesive contacts between rigid indenters and very soft transparent rubber. The softness of one of the contacting bodies is the necessary pre-requisite for formation of a large contact area whose configuration can be recorded with high resolution. The instabilities at the contact boundary leading to the boundary friction are directly observable in this system and will be studied in detail. However, we will see that for the total frictional force, the area contribution is prevalent in our experiments.

Experiments have been conducted using the setup depicted in Fig. 11. Steel spheres having various radii of curvature were indented into a $5 \mathrm{~mm}$ thick layer of transparent rubber TARNAC CRG N3005, and subsequently pulled off or moved tangentially
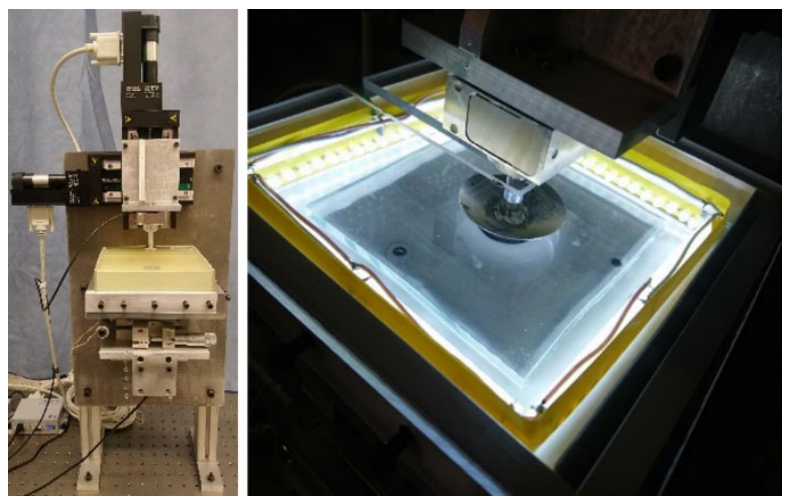

Fig. 11 Experimental setup. Left panel: general view, right panel: contact between the steel indenter and the rubber layer, with the lighting system. with precision linear stages attached to a strain gage sensor recording normal and tangential forces. The contact region was illuminated from the side with 80 LEDs and was recorded from underneath using a digital camera with the resolution of $1600 \times$ 1200 pixels. An inclination mechanism was used to ensure a parallel orientation between the rubber surface and tangential indenter movement.

\section{Normal adhesive contact}

\subsection{Flat-ended elliptic punch}

Let us start with consideration of an adhesive contact of a flat ended stamp with a face surface in form of an ellipse with an elastic flat body. Assuming that the detachment starts at the points where the stress intensity factor for the first time exceeds the critical value, it can be easily shown that the detachments should start at the ends of the major axis of the contact ellipse [29]. Numerical simulations of the detachment process confirm that detachment starts at these maximally remote from the center points and propagates inwards (Fig. 12). However,
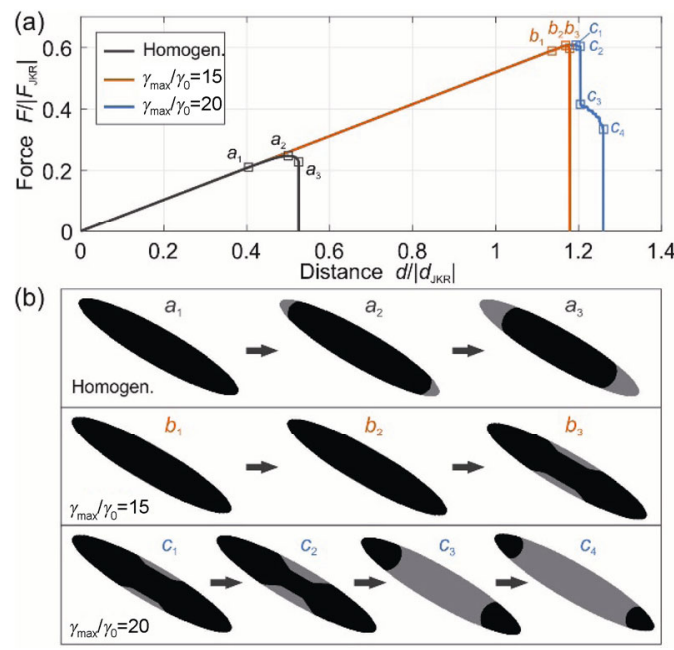

Fig. 12 Detachment process of a flat-ended punch with an elliptical face surface. (a) Three dependencies of the normal force on the distance from the plane surface (negative indentation depth); for a homogeneous system and for two heterogeneous (quadratic) distributions of the specific work of adhesion along the indenter face surface. (b) The corresponding series of contact configurations, showing the first moment when detachment starts, some intermadiate configurations, as well as the final instable configuration after which the contact is lost in a jump-like manner. 
in experiment, the detachment starts at the ends of the minor axis of the ellipse and leads to constriction of the contact in the middle of the ellipse with final separation in two not connected areas, Fig. 13.

The physical reason for this striking difference between experiment and theoretical prediction has not been clarified yet. One possible explanation could be a heterogeneity of the surface energy in the direction of the large axis of the ellipse, which could be caused e.g., by various heating of central and peripheral parts of the indenter during production process. Results of numerical simulations with artificially introduced heterogeneity of the specific work of adhesion are shown in Fig. 13. A heterogeneity can indeed lead to force-displacement relations and sequences of contact configurations very similar to those found in experiment. However, for starting detachment at the ends of the minor axis, the ratio of surface energies at the end of the ellipse and in its middle must achieve at least 13 , and for complete constriction in the middle, this ratio should be at least 20. It is hardly imaginable that such huge variations of the specific surface energies can appear due to preparation of the samples. Thus, the reason for this striking discrepancy of theory and experiment remains not clarified.

However, we would like to stress that for many

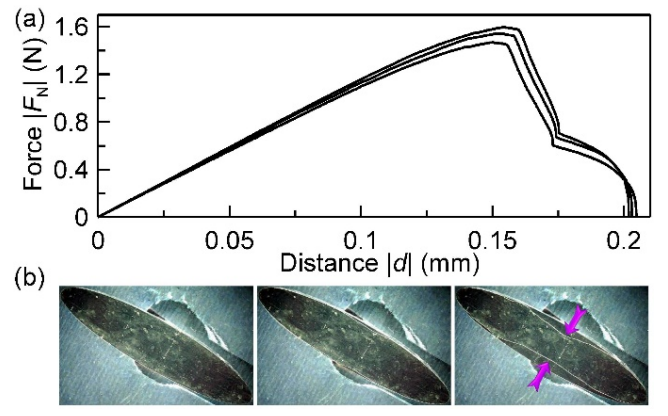

Fig. 13 Experimental observations of the detachment process of a flat-ended punch with an elliptical face surface: (a) Dependencies of the normal force on the distance from the plane surface (negative indentation depth); three curves are just three repetitions of the same experiment. (b) Contact configurations in the initial state, at the beginning of the detachment process at the ends of the minor axis, and further constriction of the contact area in the middle. Two instabilities are seen: 1) the "constriction instability" leading to a rapid division of the contact area in two separated parts, and 2) the final detachment of the two remaining contacts in the vicinity of the vertices of the ellipse. other shapes that were not as slim as those shown in Fig. 13, a good correspondence between theory and experiments has been shown (e.g., many shapes studied in Ref. [15]).

\subsection{Hysteresis of the surface energy and its time dependence}

An important question in experiments is their repeatability. Our studies of repeatability revealed a number of effects which have to be taken into account when interpreting experimental studies of adhesive contacts.

If a freshly manufactured indenter is pressed and pulled off several times, the adhesive force is significantly different in the first impression compared to the second and all subsequent ones. The reason for this effect could be chemical changes in the surface due to the first contact leading to a change of the specific surface energy after the first impression.

If a body is indented to the same depth and remains in this state for different time, it will follow different curves when it is subsequently pulled off (Fig. 14(a)). The dependence of the adhesive force on the waiting time is shown in Fig. 14(b). It can be approximated as follows:

$$
\left|F_{N, \min }\right|=0.07292 \cdot t^{0.0883}
$$

where the force is measured in Newton and time in minutes.

This means that the specific separation energy increases with the waiting time.
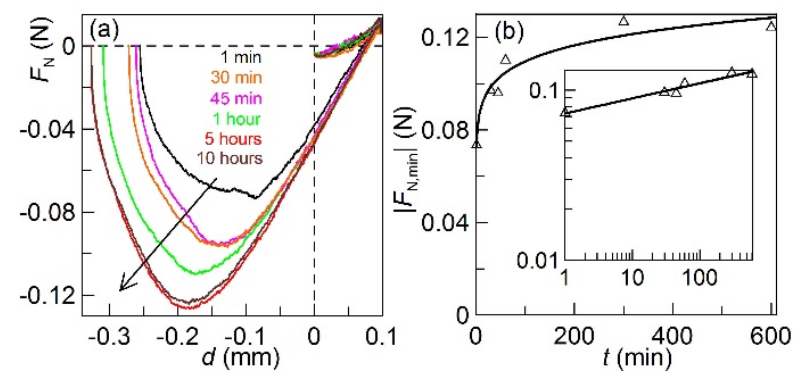

Fig. 14 (a) Dependence of the normal force on the indentation depth, for a spherical indenter with the a radius $R=40 \mathrm{~mm}$, for the soft rubber CRG N0505. Different curves correspond to different waiting time at the maximal depth of penetration. The adhesive force increases with the waiting time. (b) Dependence of the adhesive force in the pull-off phase on the waiting time. The shortest time was $t=1 \mathrm{~min}$. The inset shows the same curve on the double-logarithmic scale. 
Note that after the reversal point, all curves initially coincide and follow a linear dependence. Video recordings show that the contact area does not change during this phase. This "pinning of the boundary of contact area" is seen also in numerical simulations (Fig. 1(a)). Such behavior shows that the boundary line of the contact area is inhibited to reverse the movement immediately after reversing the loading, which means presence of a frictional force experienced by the boundary line. Such behavior has been observed and described in Ref. [44].

Before the reversal and after the linear stage, the indentation and the pull-off curves follow the theoretical JKR curve - only with different specific separation energies for the closing and opening of the contact, in correspondence with the numerical results shown in Fig. 1(b).

\section{Sliding adhesive contact}

In experiments with sliding of adhesive contacts, spherical steel indenters with radii of 11, 22, and $100 \mathrm{~mm}$ were used. They were first pressed into the rubber layer, and then were lifted up to a fixed indentation depth. We studied both positive indentation depth (represented by the value $0.2 \mathrm{~mm}$, zero indentation depth and negative "indentation depth" of $-0.015 \mathrm{~mm}$ (in the latter case, the contact does exist only due to adhesion). Subsequently, it was slowly moved in the tangential direction (typically at $v=1 \mu \mathrm{m} / \mathrm{s}$ ) over the distance $x=15 \mathrm{~mm}$. After that, the direction of the movement was reversed, and the indenter moved over the same distance $x=$ $15 \mathrm{~mm}$ back to the point of initial contact. Finally, the indenter was lifted until complete loss of contact.

The general feature of the dynamics of contact area during sliding is that it is not exactly circular (due to microscopic chemical heterogeneity and roughness). The rougher the surface (Fig. 15), the "rougher" is the contact boundary and the more discontinuous occurs its movement when increasing tangential loading. Most of the time, the boundary remains pinned by heterogeneities and the movement occurs in form of rapid jumps from one stable configuration to the other. Each jump is irreversible and during each jump, energy is lost.

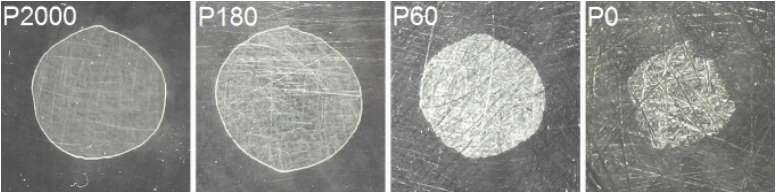

Fig. 15 Snapshots of the contact area, for different roughnesses of the indenter with radius $R=100 \mathrm{~mm}$ and zero indentation depth $d=0 \mathrm{~mm}$ during phase of detachment. The numbers P2000, P180, P60, and P0 describe the sand paper used for preparation of samples. The larger numbers correspond to smaller grain size and correspondingly to smoother surface, as listed in Table 1. The highest roughness was obtained by manual treatment with a hacksaw (P0).

Table 1 Average grain sizes of the sand paper depending on its number.

\begin{tabular}{cccc}
\hline Grain number & P2000 & P180 & P60 \\
\hline Grain size $(\mu \mathrm{m})$ & 10.3 & 82 & 269 \\
\hline
\end{tabular}

As the detailed character of the development of the contact area during sliding depends on the radius of the indenter, in the following we describe separately the results for each of the radii studied.

Indenter radius $R=22 \mathrm{~mm}$

Figure 16 shows images of the contact area during lateral displacement. Comparison of snapshots "1" and "2" shows that the displacement of the boundary of the contact area starts on the right side (front line), while it remains pinned on the left side (rear side of the contact). This behavior means that the friction force acting on the boundary line is asymmetric (smaller for propagation of the contact area and larger for shrinking).

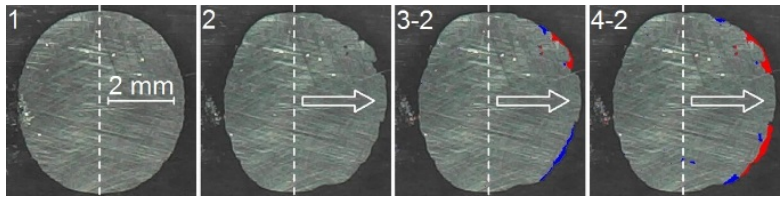

Fig. 16 Snapshots of the contact area in a contact with a spehere $(R=22 \mathrm{~mm})$. The transparent elastomer is fixed and the rough steel sphere moves to the right. The snapshot (1) corresponds to the indentation to the depth $d=0.2 \mathrm{~mm}$. The white line was inserted in the center of the contact with respect to the rigid indenter. Snapshot (2) shows the contact under subsequent displacement in the lateral direction by 0.6 $\mathrm{mm}$. The asymmetry of the contact area is seen clearly. The snapshots "3-2" and "4-2" show further evolution of the contact configuration by two subsequent displacements by 1 micrometer each. New areas formed are marked with red, and the parts which disappeared (compared to the state "2") with blue. 
Secondly, the propagation of the contact area occurs in jumps. Thus, the tangential displacement separating snapshots $3-2$ and 2 is equal to 1 micrometer, but the changes of the contact area are disproportionally large (up to the moment of the jump the boundary is pinned and does not move at all). However, the jumps of the rear part have a much smaller amplitude than those of the front area.

Let us stress that the tangential displacement can lead not only to the propagation of the front boundary in the direction of movement (red areas) but also to shrinking (moving "back", blue areas). This may be caused by a stress redistribution either due to propagation of the line in adjacent regions or due to detachment waves propagating through the contact area from the front to the rear side. These waves are not seen in the snapshots but can be easily seen in the corresponding videos.

At large indentation depth, the contact size in the direction perpendicular to the direction of sliding, tangential force as well as tangential stress do not change substantially (Fig. 17).

An essentially different behavior is observed in the case of small or negative indentation depths. In Fig. 18, the dynamics of contact area is shown for $d_{0}=0 \mathrm{~mm}$.

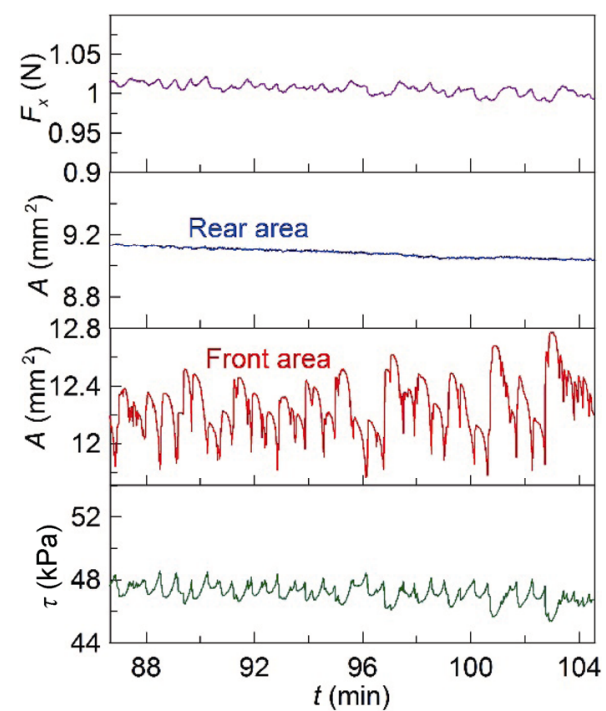

Fig. 17 A small fragment of the time dependencies of lefthand-side (rear) area (blue line in the upper part of Figure) and right-hand-side (front) area (red line in the lower part of Figure), tangential force and tangential stress in the case of $R=22 \mathrm{~mm}$ and $d=0.2 \mathrm{~mm}$.

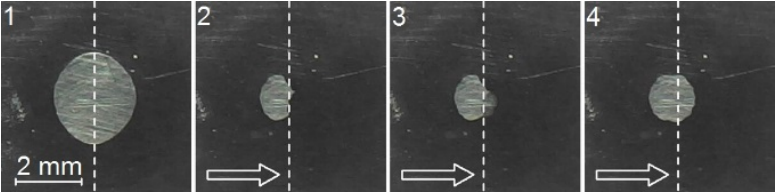

Fig. 18 Snapshots of the contact area in a contact with a sphere $(R=22 \mathrm{~mm})$ corresponding to the indentation depth $d=0 \mathrm{~mm}$. Other conditions are similar to those of Fig. 16 .

The snapshot "1" shows the contact before beginning of tangential motion, after the body has been indented to the depth $d=0.2 \mathrm{~mm}$ and moved back to the position $d=0 \mathrm{~mm}$. Snapshots $2-4$ show the contact area during tangential movement by the distance $x=0.42 \mathrm{~mm}$ at the fixed (zero) indentation depth. Finally, a stationary mode of motion is established consisting of pronounced macroscopic stick and slip phases, Fig. 19.

In this case, the difference between the dynamics of the front line and the back line of the contacts is especially pronounced. The rear contact area does not change during tangential motion (Fig. 19, blue line). The front area, on the contrary, changes vividly (Fig. 19, red line). The changes in the total area are thus mostly due to the changes in the front area. Tangential force oscillates correspondingly. However, the tangential stress $\tau$, determined as ratio of the total tangential force and the total area, remains during the sliding phase practically constant and equal to $\tau \approx 36 \mathrm{kPa}$. Detailed view provided in the right part of Fig. 19 shows what exactly happens in the contact. During the stick phase, the contact area not only does not change but also does not move, so that the force linearly increases with tangential displacement. In the subsequent sliding phase, the sliding occurs in the whole contact area at practically constant tangential stress which only weakly depends on the indentation depth (Fig. 20). This suggests that in the system studied experimentally, the main contribution to the force of friction is the area contribution.

Finally, consider the case of negative indentation depth $d=-0.015 \mathrm{~mm}$. In this case, the contact does exist only due to adhesion. The indenter was first pressed into the rubber sheet, then lifted up to $d=-0.015 \mathrm{~mm}$ (snapshot "1" in Fig. 21) and moved subsequently in tangential direction. The size of the contact decreases monotonously (in Fig. 21, 

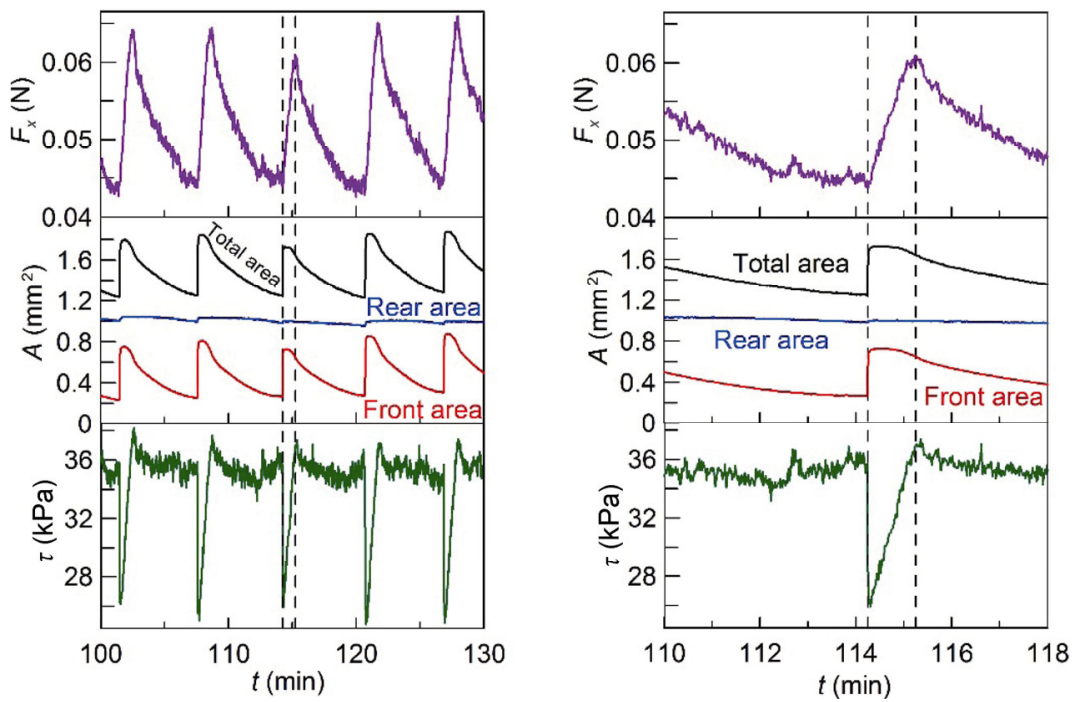

Fig. 19 Dependencies of the tangential force $F_{x}$, parts of contact areas $A$ (front area, rear area, and total area), and tangential stresses $\tau$ on time. Radius of indenter $R=22 \mathrm{~mm}$; indentation depth $d=0.0 \mathrm{~mm}$.

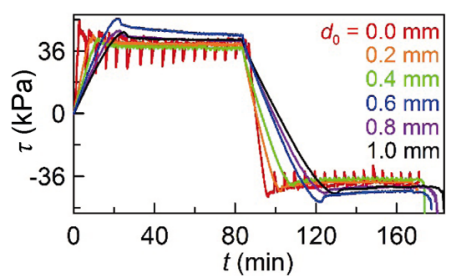

Fig. 20 Dependencies of tangential stress for forth and back movement of an indenter with radius of curvature $R=22 \mathrm{~mm}$ and 6 different indentation depths.
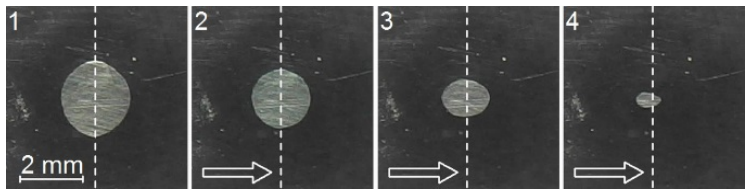

Fig. 21 Snapshots of the contact area in a contact with a sphere $(R=22 \mathrm{~mm})$, corresponding to the indentation depth $d=$ $-0.015 \mathrm{~mm}$. Other conditions are similar to those of Fig. 16.

snapshot "2" corresponds to tangential displacement $0.0369 \mathrm{~mm}, " 3$ " corresponds to $x=0.0668 \mathrm{~mm}$, and "4" to $0.0768 \mathrm{~mm})$. Finally the contact gets lost.

Figure 22 presents the normal and tangential forces, as well as the normal pressure and the tangential stress, during all of the essential stages of the experiment. The indenter was first pressed up to the depth $0.2 \mathrm{~mm}$, then lifted up to $d=-0.015$ $\mathrm{mm}$, and subsequently moved tangentially. The tangential force first increases and then decreases, and eventually vanishes (Fig. 22(b)). At the same time, the normal pressure vanishes too. This is due to the above-described decrease of the contact area until the contact completely disappears.
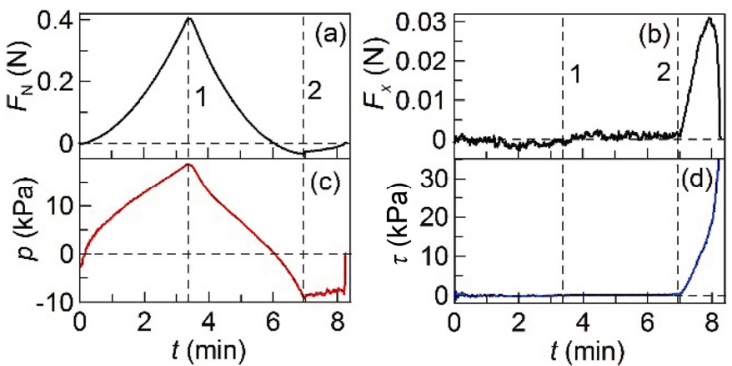

Fig. 22 Dependencies of: (a) the normal force, $F_{\mathrm{N}}$, (b) the tangential force $F_{x}$, (c) the normal mean pressure $p=F_{\mathrm{N}} / A$, and (d) the tangential stress $\tau=F_{x} / A$ on time $t$. Radius of indenter $R=22 \mathrm{~mm}$; indentation depth $d=-0.015 \mathrm{~mm}$.

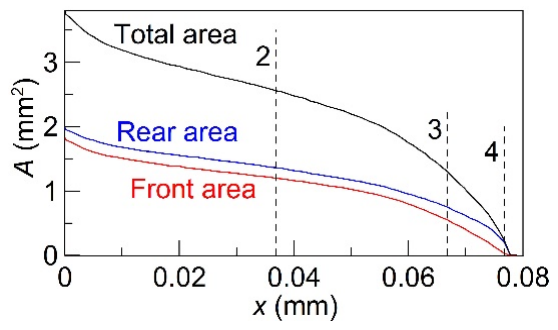

Fig. 23 Dependencies of the rear, front, and total area $A$ on the tangential displacement $x$. Radius of indenter $R=22 \mathrm{~mm}$, indentation depth $d=-0.015 \mathrm{~mm}$. Vertical lines correspond to the configurations 2, 3, and 4 in Fig. 21.

\section{Indenter radius $R=100 \mathrm{~mm}$}

To illustrate the diversity of modes observed in experiments with sliding adhesive contacts, we report also results of experiments with an indenter having the curvature radius $R=100 \mathrm{~mm}$. While from the theoretical viewpoint, the behavior of 
sliding indenters with various curvature radii should be qualitatively similar, experiments reveal several qualitative differences.

Figure 24 shows snapshots of 4 consecutive contact configuration after indentation by $d_{0}=0.2 \mathrm{~mm}$ and subsequent tangential displacement. The left snapshot corresponds to some initial tangential displacement and serves as reference for the next three, where the newly appeared contact regions are highlighted with red and the parts which disappeared with blue.

Already a study of this sequence shows that now there are large "jumps" of the contact area in the rare part of the contact (distinctively seen in Fig. 25, upper graph) while the front part moves more continuously.
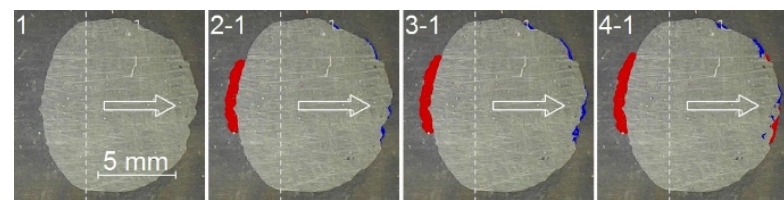

Fig. 24 Snapshots of contact configurations of a spherical indenter with radius $R=100 \mathrm{~mm}$ after indentation by $d=$ $0.2 \mathrm{~mm}$ and subsequent tangential displacement with fixed indentation depth. Areas highlighted with red are newly appeared contact regions (compared with the first snapshot which is used as reference; with blue are marked the regions which disappeared compared with the reference configuration).

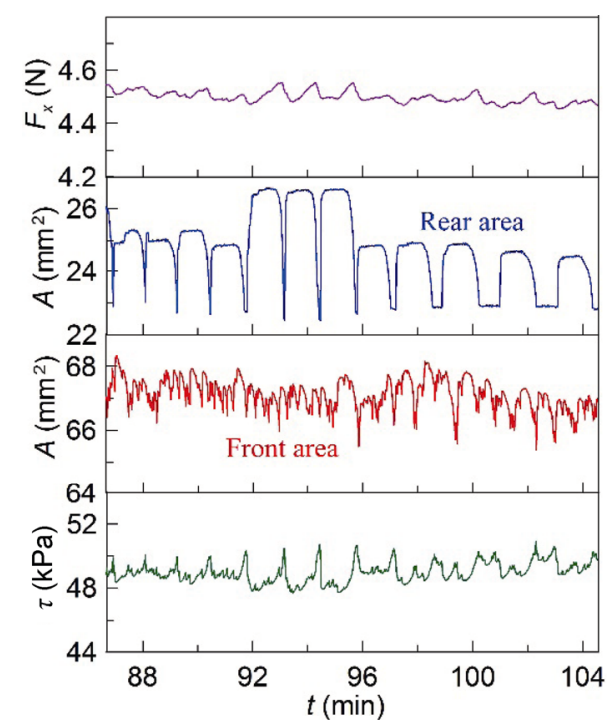

Fig. 25 Dependencies of the rear and front contact areas, tangential force and tangential stress on time $t$ in the "stationary" sliding mode, for $R=100 \mathrm{~mm}$ and $d=0.2 \mathrm{~mm}$.
Depending on the size of the indenter and loading parameters, also more complicated regimes have been observed. In some cases, the contact area in the front line jumped several times forth and back (in spite of unidirectional movement of the indenter). Attentive studies of such regimes always reveal interface waves propagating from the front side of the contact towards rear part.

As in the case of the indented with $R=22 \mathrm{~mm}$, decreasing of the indentation depth leads to appearance of a pronounced "inverted stick-slip" accompanied by reduction or complete disappearance of the front part of the contact area (Fig. 26). The tangential stress during sliding phase changes only very weakly around the value around $35 \mathrm{kPa}$.

\section{Discussion and conclusions}

This paper focuses on the tangential movement of adhesive contacts by rolling or sliding. We also studied the properties of the normal adhesive contacts since those are directly related to the energy dissipation and thus appearance of friction. Investigations have been carried out experimentally by direct observations of the contact configuration, and numerically using the FFT-based BEM for adhesive contacts.

Our studies suggest the following general picture:

1) Apparently, there exist two main contributions to friction in adhesive contacts: one coming from the contact area and the second one coming from

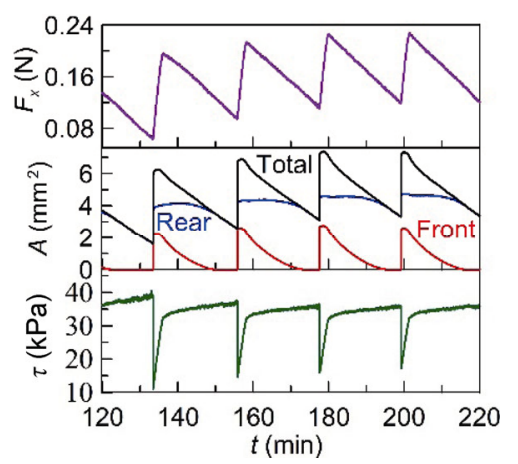

Fig. 26 Dependencies of the tangential force $F_{x}$, components of the contact areas $A$ (rear, front, and total), and mean tangential stress $\tau$ on time. Radius of the indenter $R=100 \mathrm{~mm}$, indentation depth $d_{0}=0.0 \mathrm{~mm}$. 
the boundary of the contact zone.

$$
\begin{aligned}
& F_{\text {Friction }}=F_{\mathrm{B}}+F_{\mathrm{C}} \\
& F_{\text {Friction }}=q D+A_{\text {real }} \tau_{0}
\end{aligned}
$$

where $D$ is the width of the contact facing the direction of motion, $\tau_{0}$ is a constant having dimension of stress and $q$ is a constant having dimension of linear force density, see Eq. (11). For pure rolling contact, $F_{\mathrm{C}}=0$.

When the contact is relatively circular and compact, with contact radius $a$, then $D=2 a$ and $A_{\text {real }} \approx A=\pi a^{2}$. The above statement can then be expressed as

$$
F_{\text {Friction }}=2 q \sqrt{A / \pi}+A \tau_{0}
$$

This means that formally calculated mean tangential stress in the contact is equal to

$$
\tau_{\text {mean }}=2 q(\pi A)^{-1 / 2}+\tau_{0}
$$

and increases with decreasing contact area. This seems to be supported by our experimental data (see e.g., Fig. 26).

Equation (18) implies that the boundary contribution will prevail in very small contacts, especially if they are smooth and rigid, while the area contribution is governing in large and soft contacts.

2) The boundary friction determines not only the boundary contribution to the force of friction but also the adhesive hysteresis in normal contacts. Depending on the value of the Johnson parameter $\alpha$, we can distinguish between "smooth" contacts having a more or less compact contact area and identifiable contact boundary, and "rough" contacts consisting of clouds of disconnected contact spots. For "smooth" contacts $\alpha>0.6$, the force-indentation relation can be described well by the JKR theory, but with two different specific works of adhesion, $\gamma_{1}$ and $\gamma_{2}$ for the indenting and pull-off phases. When reversing the direction of loading, the contact area remains constant and the force-distance relation is linear until the system completes the transition from one JKR curve to the other. The effective surface energies $\gamma_{1}$ and $\gamma_{2}$ are determined by the Johnson parameter, $\gamma_{1}=\gamma_{0} \cdot f_{1}(\alpha)$ and $\gamma_{2}=\gamma_{0} \cdot f_{2}(\alpha)$.

3) The physical nature of the boundary friction lies in microscopic instabilities of the contact configuration. Both numerical simulations and experiment show that tangential movement - sliding as well as rolling - is realized in both continuous movements and instable jumps. Continuous displacements are reversible, energy is dissipated solely during jumps, thus leading to appearance of macroscopic friction. This is valid even in the very rough contacts which do not show any noticeable adhesion.

4) We found that there are two main types of dynamics of the contact area: either "macroscopically continuous" (but microscopically jump-like) movement or as a pronounced macroscopic "inverted stickslip" behavior. The continuous mode is realized for small contacts and/or large indentation depth and the "stick-slip"-like mode for large contacts or small (in particular, zero or negative) indentation depths. The reason for macroscopic instabilities are apparently interfacial waves, which are observed in all cases of pronounced macroscopic instabilities (on the scale much larger than that of roughness).

5) The above properties have important macroscopic implications. In a sense, one can say that the adhesive contacts (even in the case when the adhesion is not visible because the adhesion force vanishes) are the most time in a "stick" state and move only due to rapid instable changes of the contact boundary or due to interfacial waves. This can explain a paradox described in Ref. [45]. In that study, it was experimentally found that the response of a sliding elastomer contact to dynamic loading is as if the contacts were sticking.

6) Rolling and sliding of circular contacts lead to a change of the shape in the contact. However, the change is more complex than discussed in Ref. [46]. Contacts may be "compressed", either in the sliding direction or perpendicular to it, depending on the size, indentation depth, and possibly other parameters.

7) Experiments with very slim indenters revealed a fundamental discrepancy between predictions based on the energetic detachment criterion (the JKR theory). The physical reason for this discrepancy remains not understood but is of utmost importance for the correct physical understanding of the detachment criterion and for numerical simulations of adhesive contacts. 


\section{Acknowledgements}

This work has been conducted under partial financial support from German Research Foundation (DFG) (Grant No. PO 810/55-1), the Tomsk State University Academic D.I. Mendeleev Fund Program, and the German ministry for research and education (BMBF) (Grant No. 13NKE011A).

The authors acknowledge valuable discussion with A.E. Filippov.

Contributions of authors: V.L. POPOV designed the concept of the paper, made analytical theory, carried out analysis of numerical and experimental data and drafted the manuscript. I. LYASHENKO and R. POHRT designed and built the experimental setup. I. LYASHENKO carried out experiments and processed experimental data. Q. LI, I. LYASHENKO, and R. POHRT contributed to the development of the BEM program. Q. LI executed the numerical simulations. All authors contributed equally to the editing and reviewing of the manuscript.

Open Access This article is licensed under a Creative Commons Attribution 4.0 International License, which permits use, sharing, adaptation, distribution and reproduction in any medium or format, as long as you give appropriate credit to the original author(s) and the source, provide a link to the Creative Commons licence, and indicate if changes were made.

The images or other third party material in this article are included in the article's Creative Commons licence, unless indicated otherwise in a credit line to the material. If material is not included in the article's Creative Commons licence and your intended use is not permitted by statutory regulation or exceeds the permitted use, you will need to obtain permission directly from the copyright holder.

To view a copy of this licence, visit http://creativecommons.org/licenses/by/4.0/.

\section{References}

[1] Johnson K L, Kendall K, Roberts A D. Surface energy and the contact of elastic solids. Proc R Soc Lond A 324: 301-313 (1971)

[2] Derjaguin B V, Muller V M, Toporov Y P. Effect of contact deformations on the adhesion of particles. $J$ Colloid Interface Sci 53(2): 314-326 (1975)

[3] Tabor D. Surface forces and surface interactions. $J$ Colloid Interface Sci 58(1): 2-13 (1977)

[4] Autumn K, Liang Y A, Hsieh S T, Zesch W, Chan W P, Kenny T W, Fearing R, Full R J. Adhesive force of a single gecko foot-hair. Nature 405(6787): 681-685 (2000)

[5] Popov V L, Filippov A E, Gorb S N. Biological microstructures with high adhesion and friction. Numerical approach. Physics-Uspekhi 59(9): 829-845 (2016)

[6] Heß M. A simple method for solving adhesive and non-adhesive axisymmetric contact problems of elastically graded materials. Int J Eng Sci 104: 20-33 (2016)

[7] Carbone G, Pierro E, Recchia G. Loading-unloading hysteresis loop of randomly rough adhesive contacts. Phys Rev E 92(6): 062404 (2015)

[8] Popov V L, Dimaki A V. Friction in an adhesive tangential contact in the Coulomb-Dugdale approximation. $J$ Adhesion 93(14): 1131-1145 (2017)

[9] Goryacheva I G, Makhovskaya Y Y. Elastic contact between nominally plane surfaces in the presence of roughness and adhesion. Mech Solids 52(4): 435-443 (2017)

[10] Makhovskaya Y Y., Adhesion interaction of elastic bodies with regular surface relief. Mech of Solids, 55(7):1105-1114 (2020)

[11] Pepelyshev A, Borodich F, Galanov B, Gorb E, Gorb S. Adhesion of soft materials to rough surfaces: Experimental studies, statistical analysis and modelling. Coatings 8(10): 350 (2018)

[12] Liu S B, Wang Q, Liu G. A versatile method of discrete convolution and FFT (DC-FFT) for contact analyses. Wear 243(1-2): 101-111 (2000)

[13] Pohrt R, Li Q. Complete boundary element formulation for normal and tangential contact problems. Phys Mesomech 17(4): 334-340 (2014)

[14] Khajeh Salehani M, Irani N, Müser M H, Nicola L. Modelling coupled normal and tangential tractions in adhesive contacts. Tribol Int 124: 93-101 (2018)

[15] Popov V L, Pohrt R, Li Q. Strength of adhesive contacts: Influence of contact geometry and material gradients. Friction 5(3): 308-325 (2017)

[16] Sahli R, Pallares G, Ducottet C, Ben Ali I E, Al Akhrass S, Guibert M, Scheibert J. Evolution of real contact area under shear and the value of static friction of soft materials. PNAS 115(3): 471-476 (2018)

[17] Viswanathan K, Sundaram N K. Distinct stick-slip modes in adhesive polymer interfaces. Wear 376-377: 1271-1278 (2017)

[18] Papangelo A, Scheibert J, Sahli R, Pallares G, Ciavarella M. Shear-induced contact area anisotropy explained by 
a fracture mechanics model. Phys Rev E 99(5): 053005 (2019)

[19] Ciavarella M, Cricrì G. On the application of fracture mechanics mixed-mode models of sliding with friction and adhesion. Bioinspir Biomim 15(1): 015003 (2019)

[20] Scheibert J, Sahli R, Peyrard M. Onset of sliding of elastomer multicontacts: Failure of a model of independent asperities to match experiments. Front Mech Eng 6: 18 (2020)

[21] Popov V L. Adhesive contribution to friction. AIP Conf Proc 2167(1): 020286 (2019)

[22] Heß M, Popov V L. Voltage-induced friction with application to electrovibration. Lubricants 7(12): 102 (2019)

[23] Prandtl L. Ein Gedankenmodell zur kinetischen Theorie der festen Körper. Z Angew Math Mech 8(2): 85-106 (1928)

[24] Popov V L, Gray J A T. Prandtl-Tomlinson model: History and applications in friction, plasticity, and nanotechnologies. Z Angew Math Mech 92(9): 683-708 (2012)

[25] Popov V L, Gray J A T. Prandtl-Tomlinson model: a simple model which made history. In The history of theoretical, material and computational mechanicsmathematics meets mechanics and engineering. Stein E, Ed. Berlin: Springer Berlin Heidelberg, 2014: 153-168.

[26] Hirano, Shinjo. Atomistic locking and friction. Phys Rev B Condens Matter 41(17): 11837-11851 (1990)

[27] Socoliuc A, Bennewitz R, Gnecco E, Meyer E. Transition from stick-slip to continuous sliding in atomic friction: Entering a new regime of ultralow friction. Phys Rev Lett 92(13): 134301 (2004)

[28] Dienwiebel M, Verhoeven G S, Pradeep N, Frenken J W M, Heimberg J A, Zandbergen H W. Superlubricity of graphite. Phys Rev Lett 92(12): 126101 (2004)

[29] Pohrt R, Popov V L. Adhesive contact simulation of elastic solids using local mesh-dependent detachment criterion in Boundary Elements Method. FU Mech Eng 13(1): 3-10 (2015)

[30] Li Q, Argatov I, Popov V L. Onset of detachment in adhesive contact of an elastic half-space and flat-ended punches with non-circular shape: Analytic estimates and comparison with numeric analysis. J Phys D: Appl Phys 51(14): 145601 (2018)

[31] Argatov I, Li Q, Pohrt R, Popov VL. JohnsonKendall-Roberts adhesive contact for a toroidal indenter. Proc Math Phys Eng Sci 472(2191): 20160218 (2016)

[32] Popova E, Popov V L. Note on the history of contact mechanics and friction: Interplay of electrostatics, theory of gravitation and elasticity from Coulomb to Johnson-Kendall-Roberts theory of adhesion. Phys Mesomech 21(1): 1-5 (2018)

[33] Popov V L, Heß M, Willert E. Handbook of Contact Mechanics. Exact Solutions of Axisymmetric Contact Problems. Berlin, Heidelberg: Springer Berlin Heidelberg, 2019.

[34] Johnson K L. The adhesion of two elastic bodies with slightly wavy surfaces. Int $J$ Solids Struct 32(3-4): 423-430 (1995)

[35] Popov V L. Adhesion hysteresis due to chemical heterogeneity. Preprints, 2020030131 (2020). DOI 10.20944/preprints202003.0131.v1.

[36] Li Q, Pohrt R, Popov V L. Adhesive strength of contacts of rough spheres. Front Mech Eng 5:7 (2019)

[37] Beicker K, O’Brien III E T, Falvo M R. Superfine R. Vertical light sheet enhanced side-view imaging for AFM cell mechanics studies. Sci Rep 8(1): 1504 (2018)

[38] Popov V L. Contact Mechanics and Friction. Physical Principles and Applications. 2nd edn. Berlin: Springer, 2017.

[39] Kendall K. Rolling friction and adhesion between smooth solids. Wear 33(2): 351-358 (1975)

[40] Goryacheva I G, Makhovskaya Y Y. Adhesive resistance in the rolling of elastic bodies. $J$ Appl Math Mech 71(4): 485-493 (2007)

[41] Goryacheva I, Makhovskaya Y. A model of the adhesive component of the sliding friction force. Wear 270(9-10): 628-633 (2011)

[42] Goryacheva I, Makhovskaya Y. Combined effect of surface microgeometry and adhesion in normal and sliding contacts of elastic bodies. Friction 5(3): 339-350 (2017)

[43] Popov V L. Adhesive hysteresis and rolling friction in rough "Non-adhesive" contacts. Preprints, 2020050210 (2020). DOI 10.20944/preprints202005.0210.v1.

[44] Waters J F, Guduru P R. Mode-mixity-dependent adhesive contact of a sphere on a plane surface. Proc $R$ Soc A 466(2117): 1303-1325 (2010)

[45] Nakano K, Kawaguchi K, Takeshima K, Shiraishi Y, Forsbach F, Benad J, Popov M, Popov V L Investigation on dynamic response of rubber in frictional contact. Front Mech Eng 5: 9 (2019)

[46] Sahli R, Pallares G, Papangelo A, Ciavarella M, Ducottet C, Ponthus N, Scheibert J. Shear-induced anisotropy in rough elastomer contact. Phys Rev Lett 122(21): 214301 (2019) 


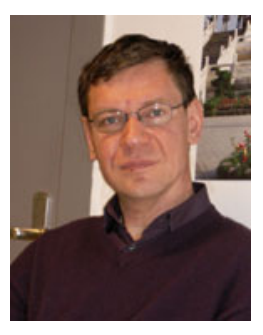

Valentin L. POPOV. He is a full professor at the Technische Universität Berlin. He studied physics and obtained his doctorate in 1985 from the Moscow State Lomonosov University. In 1985-1998, he worked at the Institute of Strength Physics and Materials Science of the Russian Academy of Sciences and was a guest professor in the field of theoretical physics at the University of Paderborn (Germany) from 1999 to 2002. Since 2002, he has been the head of the Department of System Dynamics and the Physics of Friction at the Berlin University of Technology. He has published over 300 papers in leading international journals and is the author of the book Contact Mechanics and Friction: Physical principles and applications which appeared in nine editions in German, English,

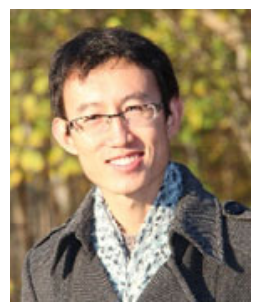

Qiang LI. He is a postdoctoral researcher at the Berlin University of Technology. He studied mechanical engineering in East China University of Science and Technology. He obtained his doctorate at the Berlin University of Technology in 2014 and now works as a scientific researcher at

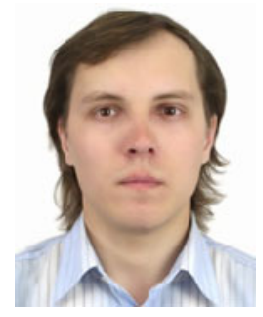

Iakov A. LYASHENKO. He is a researcher at the Berlin University of Technology and a full professor at the Sumy State University (SSU), Ukraine. He studied physical and biomedical electronics and obtained his doctorate in 2008 from the

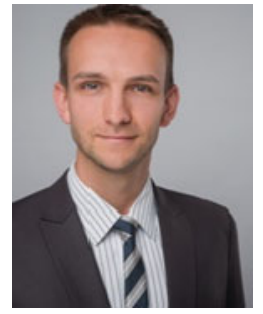

Roman POHRT. He is a researcher at the Berlin University of Technology. He studied physical engineering science with special focus on simulation and optimization of discrete and continuous problems. Since he joined the group of Prof. V. POPOV in 2010, he has been conducting experimental and numerical research on a variety of tribology related industry problems. In his Ph.D. thesis, he focussed on linking scales in the elastic
Chinese, Russian, and Spanish. He is the member of editorial boards of many international journals and is the organizer of more than 20 international conferences and workshops over diverse tribological themes. Prof. POPOV is an Honorary Professor of the Tomsk Polytechnic University, of the East China University of Science and Technology, and of the Changchun University of Science and Technology, and the Distinguished Guest Professor of the Tsinghua University. His areas of interest include tribology, nanotribology, tribology at low temperatures, biotribology, the influence of friction through ultrasound, numerical simulation of contact and friction, research regarding earthquakes, as well as topics related to materials science such as the mechanics of elastoplastic media with microstructures, strength of metals and alloys, and shape memory alloys.

the Department of System Dynamics and the Physics of Friction headed by Prof. V. L. POPOV. He has published over 50 papers in international journals including Physical Review Letters. His scientific interests include tribology, elastomer friction, hydrodynamic lubricated contact, numerical simulation of frictional behaviors, fast numerical method based on boundary element method, and adhesion.

Sumy State University. He joined the group of Prof. V. POPOV in 2014. He has published over 70 papers in international journals. His areas of interest include boundary friction, adhesion, contact mechanics, nanostructuring burnishing, dynamical systems, phase transitions, and fluctuations.

contact of fractal rough surfaces, for which he was awarded by the German Tribological Society in 2013. He has authored a series of influential papers on different tribological problems, applying and extending state-of-the-art numerical methods. $\mathrm{He}$ is the Chief-Editor of the Journal Frontiers in mechanical engineering I Tribology. His areas of interest include contact mechanics, adhesion, rail- wheelinteraction of trains, manufacturing technology, lubrication, and more generally the influence of surface topography on tribological phenomena. 